\title{
Analysis of Linear Arrays Focused in the Fresnel Region'
}

\author{
Pietro P. Lombardini, Richard Doviak, and Julius Goldhirsh ${ }^{2}$
}

\begin{abstract}
The Moore School of Electrical Engineering, University of Pennsylvania, Philadelphia, Pa.
\end{abstract}
(Received November 17, 1964; revised December 24, 1964)

\begin{abstract}
Several pertinent parameters such as gain, focal efficiency, depth and width of focus are reviewed for a near field focused linear array of radiators of arbitrary spacing. An analytic expression of the field distribution in the focal region of a phased linear array of Hertzian dipoles having a constant separation, $d$, is derived and characterized by the aforementioned parameters. A dipole spacing condition in terms of the parameters of the array is derived for which the array of discrete radiators may be approximated by a line source. Several examples of arrays consisting of more general radiators separated by Fresnel distance are considered (using a digital computer) and curves are presented comparing various array configurations with the analytic expression of a focused spherical basin.
\end{abstract}

\section{Introduction}

The field distribution in the region of the focus of apertures has received extensive investigation. Some of the original investigators were Airy [1834], Lommel [1886], Guoy [1890], and Debye [1909], who considered problems relative to the intensity distribution of visible light. More recently other investigators such as Mathews and Cullen [1956] and Sherman [1962] have considered the problem of microwave focusing. Few investigators, however, have considered the problem of characterizing the field distribution in the region of the focus of phased arrays of discrete radiators. Ricardi, in 1963, specifically described (with the use of a computer) the gain and spot size of such a linear array of Hertzian dipoles placed $\lambda / 2$ apart.

In the present work the concepts of gain in the focal region, focal efficiency, and depth and width of focus are reviewed for arrays with arbitrary spacing. A specific configuration of a focused array of Hertzian dipoles having constant separation is examined, and a separation condition is defined within which the array of discrete radiators may be approximated by a line source. Utilizing this condition, the field expression in terms of a sum of field contributions due to the individual dipoles is approximated by an integral. The integral is of identical form to that obtained by directly evaluating the field due to the line source, except that the former expression is in terms of the array parameters. Making use of the analytic field expression, the above-mentioned focal parameters are characterized in terms of the dipole spacing, height of focus, length of array, and wavelength. The "depth of focus" is specifically characterized as a function of the focal height and the region is determined within which two half-power density points exist and outside of which only one such point may be defined.

The field distribution in the focal region of several focused array configurations consisting of more general radiators separated by Fresnel distances are

${ }^{1}$ This work has been supported by the Raytheon Company as a 1964 independent research program.

${ }^{2}$ The authors are consultants to the Raytheon Co., Space and Information Systems Division, Sudbury, Mass. examined. For these examples the field is numerically obtained by directly summing (with the aid of a digital computer) the contributions due to the individual dipoles. Curves are presented comparing the field distributions along several axes with the field distribution for a spherical basin. The field intensity of the side lobes is also examined for the various array configurations for purposes of providing a measure of the relative focal efficiencies.

\section{General Considerations}

It is the purpose of the present section to review such pertinent parameters as gain, focal efficiency, depth of focus, and width of focus for a linear array of radiators. These parameters simultaneously characterize the field distribution in the focal region as well as the ability of the array to focus power efficiently.

Consider a linear array of Hertzian dipoles situated along the $z$-axis (fig. 1), each separated by a distance greater than $\lambda / 2$ but otherwise arbitrarily spaced.

For purposes of simplicity, the orientation of the dipoles will be assumed such that their axes are orthogonal to the $x$-z plane. Let $p\left(x^{\prime}, y^{\prime}, z\right)$ be the point at which a knowledge of the total field components is desired. Assume the polar parameters, $\rho$ and $\varphi$, to be such that,

$$
\rho^{2}>>\left|z-z_{p}\right|^{2} ; \quad-\frac{\pi}{4}<\varphi<\frac{\pi}{4} \text { for all possible } p,
$$

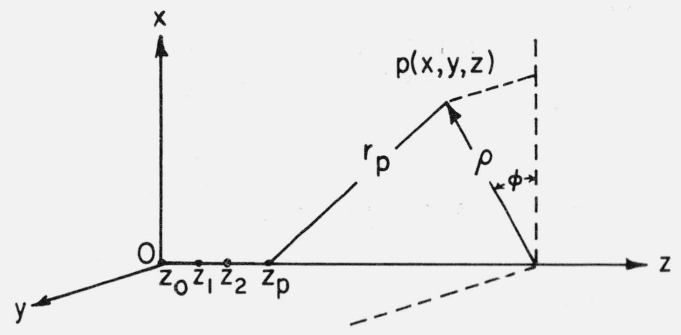

FigURE 1. Linear array configuration. 
where

$\rho=\left(x^{2}+y^{2}\right)^{1 / 2}$,

$z_{p}=$ the axial distance from the origin to the $p$ th dipole,

$\varphi=$ polar angle as shown in figure 1 .

The electric and magnetic field components due to the radiating array in the far field of the individual radiators are approximately given by

$$
\begin{aligned}
& E_{\varphi} \approx \cos \varphi \sum_{p=0}^{n} B_{p} \frac{\exp \left[-j\left(\frac{2 \pi}{\lambda} r_{p}+\psi_{p}\right)\right]}{r_{p}} . \\
& H_{z} \approx \frac{E_{\varphi}}{\eta_{0}} \\
& E_{\rho} \approx E_{z} \approx H_{\rho} \approx H_{\varphi} \approx 0
\end{aligned}
$$

where

$$
\begin{aligned}
r_{p} & =\left[x^{2}+y^{2}+\left(z-z_{p}\right)^{2}\right]^{1 / 2} \\
\psi_{p} & =\text { phase of the } p \text { th dipole } \\
\eta_{0} & =\text { wave impedance of free space. }
\end{aligned}
$$

If all the radiators are equally fed, the coefficient, $B_{p}$, is given by,

$$
B_{p}=\sqrt{\frac{\eta_{0} G_{0} P_{0}}{4 \pi}}
$$

where

$P_{0}=$ the power fed into each radiator,

$G_{0}=$ maximum gain of the individual radiators.

If the radiator parameters are adjusted such that there exists a point $\left(x_{0}, y_{0}, z_{0}\right)$ at which

$$
E_{\varphi} \approx n E_{\varphi p}
$$

where $E_{\varphi_{p}}$ is the field due to the $p$ th radiator and $p$ is arbitrary, then the array may be said to be focused here. Condition (2.8) is satisfied, for example (with $\psi_{p}=$ constant), if the individual radiators are located on a circle and the field point is located at the center of the circle.

In addition, (2.8) may be approximately satisfied if the individual dipoles lie on a straight line and are phased such that

$$
\psi_{p}=2 \pi\left(m-\frac{r_{p 0}}{\lambda}\right) m=0,1,2 \ldots
$$

where

$$
r_{p 0}=\left[x_{0}^{2}+y_{0}^{2}+\left(z_{0}-z_{p}\right)^{2}\right]^{1 / 2} .
$$

Condition (2.8) may also be approximately satisfied (assuming $\psi_{p}=$ constant) if

$$
\rho>\frac{2 D^{2}}{\lambda} \text { (where } D \text { is the array length). }
$$

For this case the maximum distance between the plane and the osculating spherical surface of radius, $\rho$, is $\leqslant \lambda / 16$. Condition (2.11), which represents the standard definition of the far field of an antenna, corresponds to the degenerate case of focusing in that (2.8) is approximately satisfied at every point in which $\rho>\rho_{0}$ along the axis of the major lobe.

The power flow in the focal region is essentially cylindrically radial and is given by

$$
S_{\rho} \approx \frac{E_{\varphi}^{2}}{\eta_{0}}
$$

The gain of the array at the focal point $\left(\rho_{0}, \varphi_{0}, z_{0}\right)$ is

$$
G \approx\left(\frac{4 \pi \rho_{0}^{2} \frac{E_{\varphi}^{2}}{\eta_{0}}}{P}\right)=n G_{0},
$$

where $P=n P_{0}$.

It is observed from (2.13) that the gain at the focal point of the array is approximately given by the expression for the gain in the far field of the array assuming the conditions in (2.1) are satisfied. This statement is in agreement with the numerical results of Ricardi [1963], who has evaluated the gain for a series of focal points of a phased linear array of dipoles whose axes were alined along the $z$-axis and spaced $\lambda / 2$ apart. He has shown the gain to be essentially constant subject to the condition that the height of focus be at least larger than the array length.

The focal efficiency, defined as the ratio of the power flowing through the region dictated by the half-power density points (at the height of the focus) to the total power radiated by the dipoles, is approximately given by

$$
\epsilon_{f} \approx \frac{1}{n \eta_{0} P_{0}} \int_{A_{0}} \int E_{\varphi}^{2} d A
$$

where $A_{0}$ is defined as the area at the altitude of the focus bounded by the periphery over which

$$
S_{\rho}=0.5 S_{0}
$$

and $S_{0}$ represents the power density flow at the focal point.

Another useful parameter, $\Delta$, called the "depth of focus" may be defined as the dimension along the axis of the main lobe whose extremities connect the half-power density points. The "width of focus" $\delta$ may likewise be defined as the distance along the line orthogonal to the main lobe axis in the plane of the array and connecting the half-power density points.

The above considerations will now be utilized in the following paragraphs in which several specific cases have been examined analytically and numerically. 


\section{Focused Linear Array With Constant Separation}

An analytic expression of the field distribution in the focal region of a phased linear array of dipoles having a constant separation will presently be derived and the focal efficiency, "depth of focus," and "width of focus" will be examined.

Consider an array of length $D$ having $(2 N+1)$ Hertzian dipoles and the same configuration as assumed in the previous section. Let each of the radiators be separated a distance $d$ apart, and let the linear array be symmetrically placed about the origin (fig. 1). Let the array be phased such that the focal point is at $\left(\rho_{0}, \varphi_{0}=0, z_{0}=0\right)$.

For this case, $r_{p}$ in (2.3) is given by

$$
r_{p}=\left[\rho^{2}+(z-p d)^{2}\right]^{1 / 2},
$$

where

$$
-N \leqslant p \leqslant N
$$

and $\psi_{p}$ is given by

$$
\psi_{p}=2 \pi m-\frac{2 \pi}{\lambda}\left[\rho_{0}^{2}+(p d)^{2}\right]^{1 / 2}, m=0,1,2 \ldots
$$

Assuming $m \approx \rho_{0} / \lambda,(2.3)$ may be approximated by

$$
E_{\varphi} \approx \frac{\exp \left[-j \frac{2 \pi}{\lambda} \rho\right]}{\rho} \cos \varphi \sum_{p=-N}^{p=+N} B_{p} \exp \left[-j \xi_{p}\right]
$$

where

$-\xi_{p}=\frac{2 \pi}{\lambda}\left\{\left[\rho^{2}+(z-p d)^{2}\right]^{1 / 2}-\left(\rho-\rho_{0}\right)-\left(\rho_{0}^{2}+(p d)^{2}\right)^{1 / 2}\right\}$.

Utilizing the binominal expansion for the first and third terms in (3.4), $\xi_{p}$ becomes

$$
\xi_{p}=\frac{\pi}{\lambda}\left[\frac{(z-p d)^{2}}{\rho}-\frac{(p d)^{2}}{\rho_{0}}\right]
$$

when the conditions

$$
\frac{\pi}{\lambda}\left|\frac{(z-p d)^{2}}{\rho}-\frac{(p d)^{2}}{\rho_{0}}\right|>>\frac{\pi}{4 \lambda}\left|\frac{(z-p d)^{4}}{\rho^{3}}-\frac{(p d)^{4}}{\rho_{0}^{3}}\right|<<1
$$

are satisfied. The above conditions are elaborated upon in appendix A and it is specifically shown that the right-hand condition of (3.6) reduces to the simplified condition (A10) in the focal region defined by the $3 \mathrm{~dB}$ points of power density.

Substituting (3.5) into (3.3) and approximating the sum by the integral, $E_{\varphi}$ becomes

$$
E_{\varphi}=\frac{\exp \left[-j \frac{2 \pi}{\lambda} \rho\right]}{\rho} \cos \varphi \int_{-N}^{N} B(p) \exp [-j \xi(p)] d p
$$

where $p$ in the integrand corresponds to a continuous variable. This approximation is valid if

$$
\left|B_{p+1}-B_{p}\right|<<1
$$

and

$$
\left|\xi_{p+1}-\xi_{p}\right|<<1 .
$$

In appendix $A$ it is demonstrated that, in the focal region defined by the $3 \mathrm{~dB}$ points of power density, (3.9) reduces to the condition $d<<0.03 D$.

Completing the square within (3.5), $\xi(p)$ becomes

$$
\xi=-\frac{\pi\left(\rho-\rho_{0}\right)}{\lambda \rho_{0} \rho}\left[\left(p d+\frac{z \rho_{0}}{\rho-\rho_{0}}\right)^{2}-\frac{\rho_{0} z^{2}}{\rho-\rho_{0}}\left(1+\frac{\rho_{0}}{\rho-\rho_{0}}\right)\right] .
$$

Assuming the above conditions examined in appendix A to be valid, we obtain upon substituting (3.10) into (3.7),

$$
E_{\varphi}=C_{0} \cos \varphi \int_{t_{0}(-D / 2)}^{t_{0}(+D / 2)} B(t) \exp \left[j \frac{\pi}{2} t^{2}\right] d t
$$

where

$$
\begin{gathered}
t \equiv\left[\frac{2}{\lambda} \frac{\left(\rho-\rho_{0}\right)}{\rho_{0} \rho}\right]^{1 / 2}\left(p d+\frac{z \rho_{0}}{\rho-\rho_{0}}\right) \\
t_{0}(D / 2)=\left[\frac{2}{\lambda} \frac{\left(\rho-\rho_{0}\right)}{\rho_{0} \rho}\right]^{1 / 2}\left(\frac{z \rho_{0}}{\rho-\rho_{0}}+\frac{D}{2}\right) \\
t_{0}(-D / 2)=\left[\frac{2}{\lambda} \frac{\left(\rho-\rho_{0}\right)}{\rho_{0} \rho}\right]^{1 / 2}\left(\frac{z \rho_{0}}{\rho-\rho_{0}}-\frac{D}{2}\right) \\
C_{0}=\left[\frac{\lambda \rho_{0}}{2\left(\rho-\rho_{0}\right) \rho d^{2}}\right]^{1 / 2}\left[\exp -j \frac{2 \pi}{\lambda}\left(\rho+\frac{z^{2}}{2\left(\rho-\rho_{0}\right)}\right)\right]
\end{gathered}
$$

It should be remarked that, in passing from the summation (3.3) to the integral form (3.7), the linear array problem reduces to the focused line source problem. Thus, the resultant field is of identical form to that obtained for a focused line source, except that the solution is now in terms of the parameters of the array. The field of the line source has previously been derived as a limiting case of a focused rectangular aperture [Sherman, 1962].

For the specific case in which the dipoles are equally fed, $B_{p}=B_{0}$ [given by (2.7)], and (3.11) becomes

$$
E_{\varphi}=B_{0} C_{0} \cos \varphi\left[\left(C\left(t_{0}^{+}\right)-C\left(t_{0}^{-}\right)\right)+j\left(S\left(t_{0}^{+}\right)-S\left(t_{0}^{-}\right)\right)\right]
$$


where $C$ and $S$ are the cosine and sine Fresnel integrals, respectively. Expression (3.16) thus corresponds to the electric field in the focal region subject to the condition that (2.1), (3.6), and (3.9) are satisfied.

It is of interest to note that along the plane $z=0, E_{\varphi}$ reduces to

$$
E_{\varphi} \approx 2 C_{0} B_{0} \cos \varphi\left[C\left(t_{0}^{+}\right)+j S\left(t_{0}^{+}\right)\right]
$$

and along the cylindrical surface $\rho=\rho_{0}, E_{\varphi}$ becomes

$$
E_{\varphi} \approx B_{0} \frac{D}{\rho_{0} d} \cos \varphi \frac{\sin \left(\frac{\pi z D}{\lambda \rho_{0}}\right)}{\left(\frac{\pi z D}{\lambda \rho_{0}}\right)} \epsilon^{-j \frac{2 \pi}{\lambda}}\left[\rho_{0}+\frac{z^{2}}{2 \rho_{0}}\right] .
$$

The focal efficiency may now be obtained by substituting (3.18) into (2.14) and integrating over the cylindrical surface (at the altitude of the focus $\rho=\rho_{0}$ ) defined by the half-power density points. Hence, $\epsilon_{f}$ becomes

$$
\epsilon_{f}=7.3 \times 10^{-2}\left(\frac{\lambda G_{0}}{d}\right) .
$$

Note that the efficiency is enhanced as the dipole spacing is reduced. For the special case in which the dipoles are spaced $\lambda / 2$ apart, the efficiency becomes

$$
\epsilon=21.9 \text { percent }
$$

Since there are theoretically two focal regions (one below and the other above the array), approximately 44 percent of the total power may be said to flow through the regions defined by half-power density boundaries.

The width of focus, $\delta$, may be obtained from the relationship

$$
\frac{\sin \left(\frac{\pi z D}{\lambda \rho_{0}}\right)}{\left(\frac{\pi z D}{\lambda \rho_{0}}\right)}=0.707
$$

Hence,

$$
\delta=0.89\left(\frac{\rho_{0}}{D}\right) \lambda .
$$

The depth of focus, $\Delta$, is obtained by solving the following transcendental equation for $t_{0}$ :

$$
\left(\frac{E_{\varphi_{1}}}{E_{\varphi_{m}}}\right)^{2}=\frac{\left[1 \pm 4 \alpha t_{0}^{2}\right]^{2}}{t_{0}^{2}}\left[C^{2}\left(t_{0}\right)+S^{2}\left(t_{0}\right)\right]=\frac{1}{2}
$$

where

$$
\alpha=\frac{\rho_{0}}{\left(\frac{2 D^{2}}{\lambda}\right)}
$$

and $E_{\varphi_{1}}$ and $E_{\varphi_{m}}$ are the magnitudes of (3.16) evaluated at the half-power point and the focal point, respectively. Solving (3.23) with the $(+)$ sign gives the radial distance of the half-power point below the focus:

$$
\rho_{1}=\frac{\rho_{0}}{1+4 \alpha t_{0}^{2}(+)}
$$

and the solution with the (-) sign results in the radial distance of the half-power point above the focus:

$$
\rho_{2}=\frac{\rho_{0}}{1-4 \alpha t_{0}^{2}(-)} .
$$

The normalized depth of focus defined by

$$
\frac{\Delta}{\rho_{0}}=\frac{\rho_{2}-\rho_{1}}{\rho_{0}}=\frac{4 \alpha\left[t_{0}^{2}(+)+t_{0}^{2}(-)\right]}{\left[1+4 \alpha t_{0}^{2}(+)\right]\left[1-4 \alpha t_{0}^{2}(-)\right]}
$$

is plotted in figure 2 as a function of $\alpha$.

Since the condition (2.1) implies $\rho_{0}>D$, the curve in figure 2 is valid for

$$
\alpha>\frac{\lambda}{2 D}
$$

It may be observed for $\alpha \leqslant 3 \times 10^{-2}$,

$$
\Delta \approx 6.85\left(\frac{\rho_{0}}{D}\right)^{2} \lambda
$$

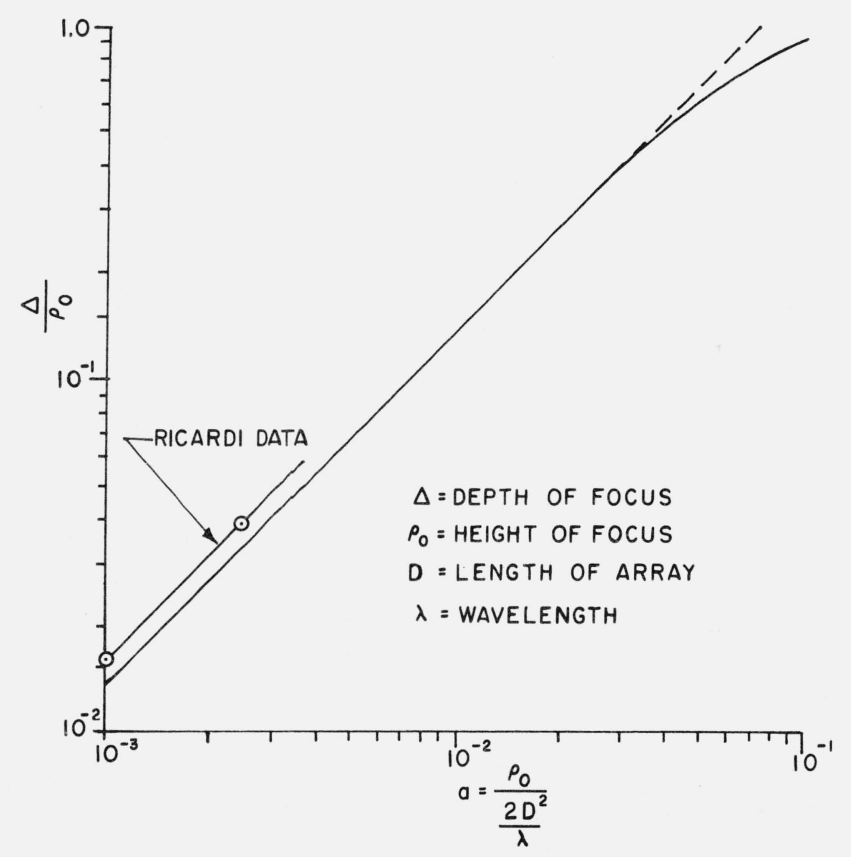

FIGURE 2. Normalized depth of focus versus normalized height of focus. 
The "focal depth-to-width" ratio is then given by

$$
\frac{\Delta}{\delta}=7.7\left(\frac{\rho_{0}}{D}\right)
$$

It may be noted that in the solving of the transcendental (3.23) for $t_{0}(+)$, no solution existed for $\alpha>0.1$. This implies that no lower half-power density point, $\rho_{1}$, exists for this range of $\alpha$. Hence, in addition to (2.8), the definition of focusing may thus be extended to satisfy the additional condition that two half-power points along the vertical direction must exist. Hence, for the present case, the focal region is limited to the region

$$
\rho<0.1\left(\frac{2 D^{2}}{\lambda}\right)
$$

It is shown in appendix A that the lower limit for $\rho$ is given by

$$
\rho^{2} \gg 0.64 D^{2}
$$

\section{Field Distribution in Focal Region of Linear Arrays Whose Radiators Âre Separated by Fresnel Distances}

In the previous section, the field distribution in the focal region was analytically characterized for a phased linear array of Hertzian dipoles separated a constant distance apart.

In the present section, the field distributions in the focal region of various linear arrays are numerically characterized for the case in which more general radiators are separated by their Fresnel distances.

\subsection{Formulation of the Problem}

Consider an array of identical radiators situated along an axis $y_{1}$ in the $x-y$ plane making an angle $\alpha$ with respect to the $x$-axis, as shown in figure 3 . It is assumed that each radiator is focused at $x=y=0$, $z=h$.

Let the field pattern of each of the radiators have an angular variation given by

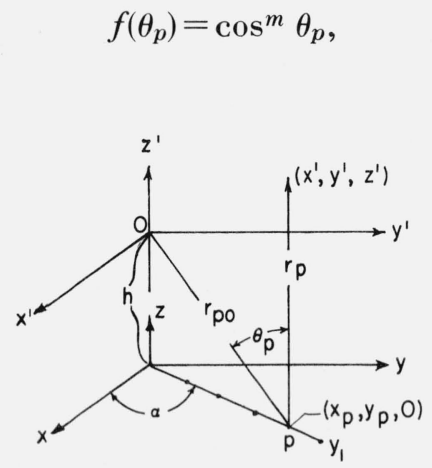

FIGURE 3. More general radiator configuration. where $m$ is an arbitrary integer and $\theta_{p}$ is the angle between the lines connecting the $p$ th radiator and the focal point 0 , and the $p$ th radiator and any other point $x^{\prime}, y^{\prime}, z^{\prime}$ (as shown in fig. 3). Assume the point $p\left(x^{\prime}\right.$, $\left.y^{\prime}, z^{\prime}\right)$ is in the far field of the individual radiators and a condition analogous to (2.1) to exist. Let the radiators be identically polarized such that the dominant component of the electric field is given by

$$
E_{y}=\sum_{p=0}^{N} \frac{A_{p}}{r_{p}} \cos ^{m} \theta_{p} \exp \left[-j\left(\frac{2 \pi}{\lambda} r_{p}+\varphi_{p}\right)\right],
$$

where

$$
r_{p}=\left[\left(x^{\prime}-x_{p}\right)^{2}+\left(y^{\prime}-y_{p}\right)^{2}+\left(h+z^{\prime}\right)^{2}\right]^{1 / 2}
$$

and

$\cos \theta_{p}=\frac{x_{p}^{2}+y_{p}^{2}+h^{2}-x^{\prime} x_{p}-y^{\prime} y_{p}+h z^{\prime}}{\left(x_{p}^{2}+y_{p}^{2}+h^{2}\right)^{1 / 2}\left[\left(x^{\prime}-x_{p}\right)^{2}+\left(y^{\prime}-y_{p}\right)^{2}+\left(h+z^{\prime}\right)^{2}\right]^{1 / 2}}$.

At the focus point, 0 ,

$$
E_{y} \approx N E_{y p}
$$

If (4.4) is to be satisfied, $d_{p}$ and $\varphi_{p}$ must be such that

$$
\frac{2 \pi}{\lambda}\left(d_{p}^{2}+h^{2}\right)^{1 / 2}+\varphi_{p}=2 \pi\left(p+\frac{h}{\lambda}\right),
$$

where $d_{p}$ corresponds to the location of the $p$ th radiator of the linear array and is given by

$$
d_{p}=\left(x_{p}^{2}+y_{p}^{2}\right)^{1 / 2} .
$$

Solving for $d_{p}$ from (4.5),

$$
d_{p}=\left\{\lambda^{2}\left(p^{2}-\frac{\varphi_{p}}{\pi} p+\frac{\varphi_{p}^{2}}{4 \pi^{2}}\right)+\lambda h\left(2 p-\frac{\varphi_{p}}{\pi}\right)\right\} .
$$

For the case in which $\varphi_{p}$ is constant, the distances, $d_{p}$, correspond to the classical Fresnel separations of diffractions theory. Substituting the parameters $d_{p}$ and $\alpha$ into (4.3), $E_{y}$ becomes

$$
\begin{aligned}
E_{y}=\sum_{p=0}^{N} A_{p} \frac{\left[d_{p}^{2}+h^{2}+h z^{\prime}-d_{p}\left(x^{\prime} \cos \alpha+y^{\prime} \sin \alpha\right)\right]^{m}}{\left[d_{p}^{2}+h^{2}\right]^{m / 2} r_{p}^{m+1}} \\
\times \exp \left[-j\left(\frac{2 \pi}{\lambda} r_{p}+\varphi_{p}\right)\right],
\end{aligned}
$$

where

$r_{p}=\left[\left(x^{\prime}-d_{p} \cos \alpha\right)^{2}+\left(y^{\prime}-d_{p} \sin \alpha\right)^{2}+\left(h+z^{\prime}\right)^{2}\right]^{1 / 2}$.

In order to increase the utility of the following numerical examples, (4.8) is expressed independently of $\lambda$ as 


$$
\begin{array}{r}
\lambda E_{y}=\sum_{p=0}^{N} A_{p} \frac{\left[a_{p}^{2}+b^{2}+b c-a_{p}(d \cos \alpha+e \sin \alpha)\right]^{m}}{\left[a_{p}^{2}+b^{2}\right]^{m / 2} r_{p}^{m+1}} \\
\times \exp \left[-j\left(\frac{2 \pi}{\lambda} r_{p}+\varphi_{p}\right)\right],
\end{array}
$$

where

$$
\begin{aligned}
d_{p} & =a_{p} \lambda, \quad z^{\prime}=c \lambda, \quad y^{\prime}=e \lambda, \\
h & =b \lambda, \quad x^{\prime}=d \lambda, \\
r_{p} & =\lambda\left[\left(d-a_{p} \cos \alpha\right)^{2}+\left(e-a_{p} \sin \alpha\right)^{2}+(b+c)^{2}\right]^{1 / 2} .
\end{aligned}
$$

\subsection{Numerical Examples}

The term $E_{y}$, given by (4.8) has been computed using a digital computer. The specific parameters chosen were $D=6 \times 10^{3} \lambda$ and $h=2.11 D$ for the following linear array configurations:

(1) $\alpha=0, m=2, N=802$ radiators, $\varphi_{p}=0$;

(2) $\alpha=0, m=2, N=1602$ radiators,

$$
\varphi_{p}=\left\{\begin{array}{l}
\varphi_{2 n}=0 \\
\varphi_{2 n+1}=\pi,
\end{array} \quad \text { where } n=0,1,2, \ldots, 800 ;\right.
$$

(3) fifty-nine alinements at $3^{\circ}$ apart (i.e., $\alpha=1.5,4.5$, $\left.7.5, \ldots, 178.5^{\circ}\right) m=2$. Each alinement contains 1602 radiators and are phased as in 2).

For the first configuration the relative field strength, $\lambda E_{y}$, is plotted (fig. 4) along the vertical axis about the region of the focus. This variation is found to coincide with the $\frac{\sin x}{x}$ distribution corresponding to a spherical basin of diameter equal to the array length. For the same configuration (fig. 5), the field distribution is plotted along the $x^{\prime}$-axis $\left(z^{\prime}=0\right)$ about the region of the focus and compared with the corresponding field distribution $\left(2 J_{1}(x) / x\right)$ for a spherical basin. In figure 6 the normalized field intensities along the $x$-axis are compared for configurations (1) and (2) over a distance of $6 \times 10^{3} \lambda$. Since the points were evaluated at $166 \lambda$ intervals, they are connected by straight lines. It may be observed that the intensity of the side lobes is considerably reduced for the case in which the alternate radiators are counterphased [configuration (2)]. This reduction of the side lobe intensity implies a greater power flow through the main lobe and hence an increase in the overall focusing efficiency. This conclusion is also consistent with the result (3.19) which also indicates an increase in efficiency as the radiator distance is reduced.

Note that at several locations the differences in normalized field intensities between the two configurations are greater than $20 \mathrm{~dB}$. Also plotted in figure 6 is the normalized field intensity as a function of lateral distance for configuration (3). It is of interest to note that the side lobe intensity is reduced considerably over the intensity for the first two configurations (i.e., approximately $10 \mathrm{~dB}$ reduction over the second configuration).

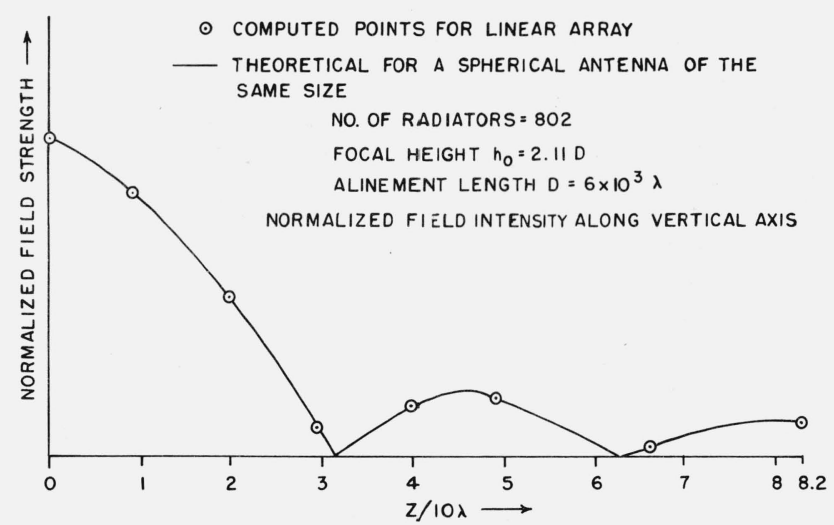

FIGURE 4. Normalized field intensity along vertical axis.

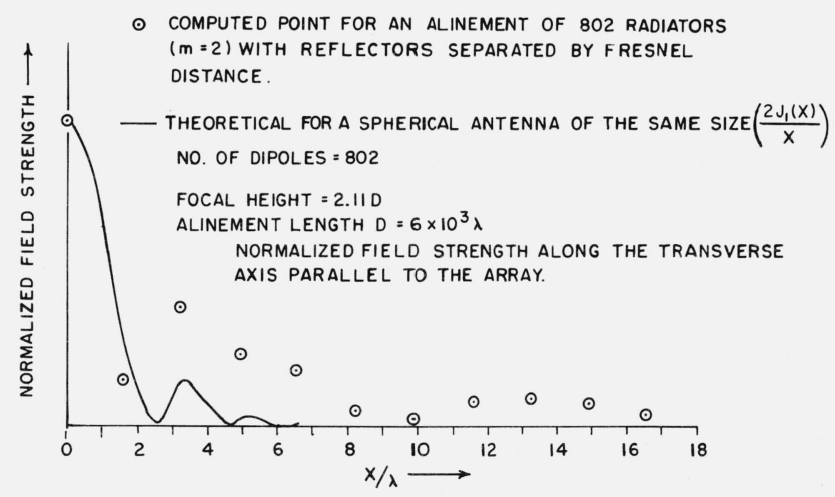

Figure 5. Normalized field strength along the transverse axis parallel to the array.

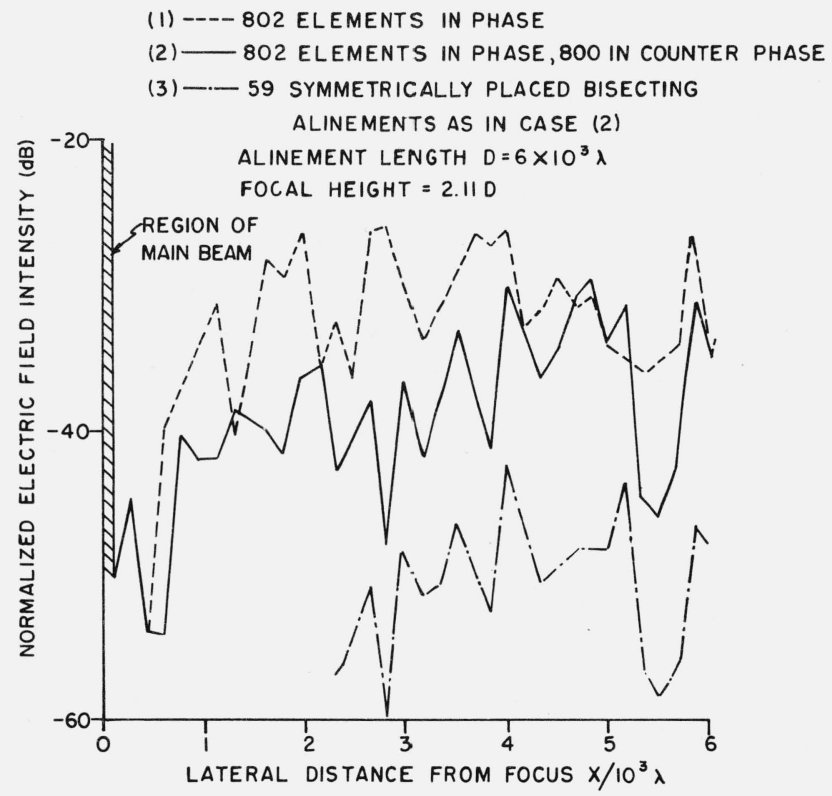

FIGURE 6. Side lobe intensities for three configurations of antennas. 


\section{Conclusions}

The gain at the focal point due to a focused linear array of identical Hertzian dipoles separated by arbitrary distances is approximately given by the product of the gain of the individual radiators and their total number. This approximation is valid if the radial distance to the field point, $\rho$, is larger than the array length, $D$ (condition A4).

For the case in which Hertzian dipoles are spaced a distance $d$ apart, the condition for which the discrete array may be approximated by a continuous line source is given by $d \ll 3 \times 10^{-2} \mathrm{D}$, (condition A9). If use is made of this approximation many of the results become analogous to those pertaining to the line source problem, except that the former results are in terms of the parameters of the focused array.

The focusing efficiency for the array of Hertzian dipoles of constant separation is proportional to the product of the gain and wavelength and inversely proportional to the dipole spacing (3.19). The total efficiency for a $\lambda / 2$ spacing is approximately 44 percent.

A limiting focal distance $\rho_{0}$ exists such that the "depth of focus," $\Delta$, beyond this distance can not be specified. That is, focusing outside this limiting region only gives rise to $3 \mathrm{~dB}$ degradation of the field intensity (along the main lobe axis) at points above the focal point but none below it. For the case in which the Hertzian dipoles are equally spaced this limiting

distance is given by $\rho_{0 L} \approx 0.1\left(\frac{2 D^{2}}{\lambda}\right)$. It may therefore

be convenient to define the region $\rho>\rho_{0 L}$ as the region for which focusing is not possible. For the region given by, $D<\rho_{0}<\rho_{0 L}$, the depth of focus varies as the ratio $\left(\frac{\rho_{0}}{D}\right)^{2}$ while the "width of focus" varies only as $\left(\frac{\rho_{0}}{D}\right),(3.29)$ and $(3.22)$, respectively.

Several interesting results become evident from the numerical examples of section 4 and the corresponding curves given in figures 4,5 , and 6 . For the case in which the radiators are in phase and separated by Fresnel distances, the field distribution along the main lobe axis coincides with the field distribution of a spherical basin antenna for $m=2$. The intensity of the side lobes is reduced by as much as $20 \mathrm{~dB}$ at several locations if additional radiators are placed in counterphase with respect to the original radiators. Further reduction occurs when additional alinements are present.

\section{Appendix A. Recapitulation and Dis- cussion of Approximations of Section 3}

In the derivation of the expression (3.11) the following approximations were made:

$$
\rho^{2}>>[z-p d]^{2}
$$

$$
\begin{aligned}
& \frac{\pi}{\lambda} \mid\left(\frac{[z-(p+1) d]^{2}}{\rho}-\frac{[(p+1) d]^{2}}{\rho_{0}}\right) \\
& -\left(\frac{(z-p d)^{2}}{\rho}-\frac{(p d)^{2}}{\rho_{0}}\right) \mid<<1 \quad \\
& \frac{\pi}{\lambda}\left|\frac{(z-p d)^{2}}{\rho}-\frac{(p d)^{2}}{\rho_{0}}\right|>>\frac{\pi}{4 \lambda}\left|\frac{(z-p d)}{\rho^{3}}-\frac{(p d)^{4}}{\rho_{0}^{3}}\right|<<1
\end{aligned}
$$

where $-N<p<N$.

Assuming the region of interest to be the focal region bounded by the half-power density periphery, more straightforward conditions may be extracted from (A1) to (A3).

Substituting (3.22) into (Al) and letting $p=-N$, and $\alpha_{\max }=0.1$, the worst case condition becomes

$$
\rho^{2}>>0.64 D^{2}
$$

Expanding and combining the terms of (A2), we obtain the condition

$$
\frac{\pi d^{2}}{\rho_{0} \lambda} \frac{(2 p+1)\left(\rho-\rho_{0}\right)}{\rho}<<1 .
$$

The worst case condition may be obtained by injecting, in the numerator of (A5), $p=+N$ and, from (3.26),

$$
\left(\rho-\rho_{0}\right)_{\max }=\frac{4 \alpha t_{0}^{2}(-) \rho_{0}}{1-4 \alpha t_{0}^{2}(-)}
$$

and in the denominator of (A5), from (3.25)

$$
\rho_{\min }=\frac{\rho_{0}}{1+4 \alpha t_{0}^{2}(+)} .
$$

Hence, (A5) becomes

$$
\left(\frac{2 \pi d}{D}\right) t_{0}^{2}(-)\left[\frac{1+4 \alpha t^{2}(+)}{1-4 \alpha t_{0}^{2}(-)}\right]<<1 \text {. }
$$

In figure $7, t_{0}(-)$ and $t_{0}(+)$ are plotted as a function of $\alpha$ [i.e., solution to (3.23)]. The worst case condition 


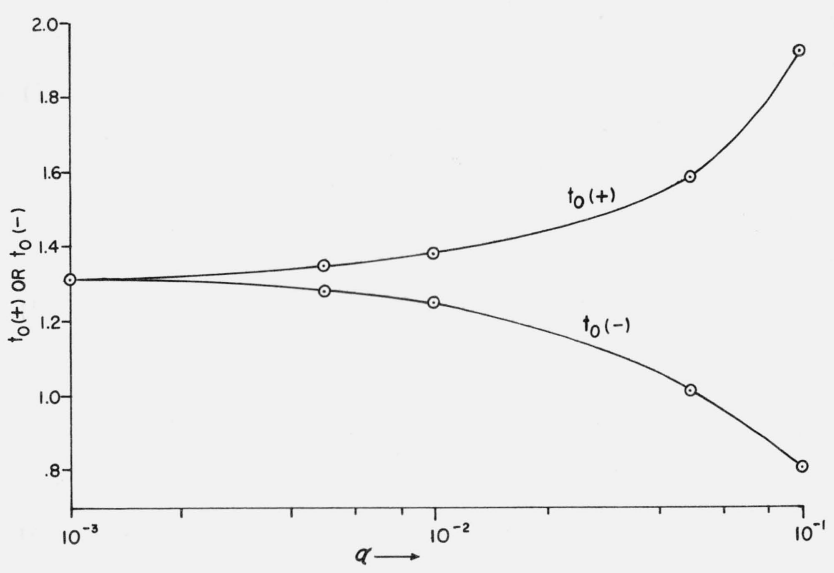

FiguRE 7. Solution of transcendental equation (3.23).

of (A8) corresponds to the maximum values of $t_{0}(-)$, $\alpha t_{0}(+)$, and $\alpha t_{0}(-)$. Extracting these values from the curve, (A8) reduces to

$$
d \ll 3 \times 10^{-2} D .
$$

Condition (A9) thus represents the dipole distance condition for which the linear array field solution takes the form of the line source solution.

Consider now the right side condition of (A3). Substituting (3.22), $p=-N$, and (A7) into this inequality, there results the further stipulation of $\rho_{0}$ that

$\rho_{0}^{2} \gg \frac{2.45 \times 10^{-2}\left(\left[1+4 \alpha t_{0}^{2}(+)\right]^{3}[1+1.78 \alpha]^{4}-1\right)}{\alpha} D^{2}$, where

$$
\alpha=\frac{\rho_{o}}{\frac{2 D^{2}}{\lambda}} .
$$

It should be remarked that the condition which must be chosen with regard to $\rho_{0}$ should be the more stringent of the conditions given by (Al) and (Al0). The left-hand side of (A3) may be shown to be satisfied if $(\mathrm{Al})$ or $(\mathrm{A} 10)$ are valid.

\section{References}

Airy, G. B. (1834), On the diffraction of a glass object with a circular aperture, Trans. Cambridge Phil Soc. 5, 283.

Debye, F. (1909), Das Verhalten von Lichtwellen in der Nähe eines Brennpunktes oder einer Brennlinie, Annalen der Physik 30, 755. Guoy, A. (1890), Sur une propriété nouvelle des ondes lumineuses, Comptes Rendus de Paris 110, 1251.

Lommel, E. (1886), De Beugungserscheinumgen einer Öffnung und eines Kreisrunden Schirmchens, Königlich Bayerischen Akademie der Wissenschaften, Abhandlung der MathematischPhysikalischen Klasse 15, 229.

Matthews, P. A., and A. L. Cullen (July 1956), A study of the field distribution at an axial focus of a square microwave lens, Institution of Electrical Engineers, Monograph No. 186R, p. 449.

Ricardi, L. J. (1963), Near-field characteristics of a linear array, Electromagnetic Theory and Antennas, p. 1301 (Macmillan Publishing Co., New York, N.Y.).

Sherman, J. W. (July 1962), Properties of focused apertures in the Fresnel region, IRE Trans. Ant. Prop. AP-10, No. 4, 399-408. 